\title{
MAI and Noise Constrained LMS Algorithm for MIMO CDMA Linear Equalizer
}

\author{
Khalid Mahmood ${ }^{1}$, Syed Muhammad Asad ${ }^{2}$, Muhammad Moinuddin ${ }^{3}$, Waqas Imtiaz \\ ${ }^{1,4}$ Iqra National Universty Peshawar, Pakistan \\ ${ }^{2}$ University of Hafr Al Batin, Kingdom of Saudi Arabia \\ ${ }^{3}$ Center of Excellence in Intelligent Engineering Systems (CEIES) King Abdul Aziz University, Jeddah
}

Kingdom of Saudi Arabia

\begin{abstract}
This paper presents a constrained least mean squared (LMS) algorithm for MIMO CDMA linear equalizer is presented, which is constrained on spreading sequence length, number of subscribers, variances of the Gaussian noise and the multiple access interference (MAI) plus the additive noise (introduced as a new constraint). The novelty of the proposed algorithm is that MAI and MAI plus noise variance has never been used as a constraint in MIMO CDMA systems. Convergence analysis is performed for the proposed algorithm in case when statistics of MAI and MAI plus noise are available. Simulation results are presented to compare the performance of the proposed constrained algorithm with other constrained algorithms and it is proved that the new algorithm has outperformed the existing constrained algorithms.
\end{abstract}

Index Terms-Least mean squared (LMS), multiple input, multiple output (MIMO), linear equalizer, multiple access interference (MAI), Variance, AWGN, adaptive algorithm

\section{INTRODUCTION}

It is shown in the literature that performance of an adaptive algorithm may be enhanced if partial knowledge of a particular channel is blended in the algorithm design[1], [2]. Based on this idea, [1] presented an algorithm (Noise-constrained LMS) for identification and tracking of finite impulse response (FIR) channels using the variance of the receiver noise. An advantage of the noise constrained least mean squared (NCLMS) is that it outperforms the LMS algorithm while keeping the same computational complexity. A complementary pair LMS (CPLMS) [3] was initiated by using constrained optimization technique named augmented Lagrangian (AL) which can be utilized to solve the problem of selecting an appropriate update step-size in LMS algorithm. This technique was utilized in [4], in which the knowledge of the variance of MAI plus noise was incorporated to develop what is called the constrained LMS algorithm (MNCLMS) for CDMA systems.

Since MAI and the additive white Gaussian noise (AWGN) effect performance of CDMA multi user, multi-antenna environment, it is imperative to design a receiver architecture which will negate the effect of MAI and additive noise. This requires a MIMO implementation of the MNCLMS algorithm presented in [4]. The proposed constrained algorithm is developed by incorporating MIMO MAI and noise variances thus resulting in a generalized MIMO MAI plus noise constrained LMS (MIMO-MNCLMS) adaptive algorithm. As it is generalized, we can deduce MAI constrained algorithm, noise constrained algorithm and zero constrained noise algorithm as special cases.

This paper is organized as follows. After introductory remarks, section 2 presents the motivation for the algorithm development. Algorithm development is presented in section 3 whereas section 4 deals with the convergence analysis. Computational complexity of the algorithm is given in section 6 . In order to find the theoretical findings, simulation results are presented in Section 7 . Concluding remarks are shown in section 8 .

\section{Motivation}

Adaptive algorithms such as LMS and RLS do not use models for channel coefficients and/or noise, whereas model based algorithms utilize various types of models such as random walk, auto-regressive etc. for coefficients and AWGN. Model parameters are either known or jointly estimated with a channel. Adaptive algorithms can be inferred to as model based algorithms with model parameters' choice dependent on data [5]. It has been reported in the literature that practically it is possible to enhance the performance of an adaptive algorithm if partial knowledge of the channel is available provided that the computational cost of an algorithm is not increased tremendously. According to the noise constrained LMS algorithm [6]

$$
\begin{gathered}
\mathbf{w}_{n+1}=\mathbf{w}_{n}+\mu_{n}^{l} e_{n} \mathbf{X}_{n} \\
\mu_{n+1}=2 \mu_{n}^{l}\left(1+\gamma \lambda_{n}\right) \\
\lambda_{n+1}=\lambda_{n}+\beta\left[\left(\frac{1}{2} e_{n}^{2}-\sigma_{v_{m}^{l}}^{2}\right)-\lambda_{n}\right]
\end{gathered}
$$

Where $\lambda, \alpha$ and $\beta$ are positive constants. This is a variable step size (LMS) algorithm because step size rule is applicable due to the constraint on the noise variance. The computational cost of the aforementioned algorithm is the same as of LMS but the convergence rate of the noise constrained LMS algorithm is much faster than the LMS due to its three independent parameters. 
MAI is the major limiting factor in the system performance of a multiuser environment, it is required to design a multi receiver scheme which will negate the effect of MAI and the additive noise. Previous research work treated MAI as a part of interfering noise. This assumption is not practically correct which led this work to use MAI as an additional constraint by using structured information contained in it. We also believe that by using the combined information of MAI and the interfering noise to form into a single constraint would result in an algorithm which would outperform the noise only constrained algorithm. It is worth mentioning here that by using MAI alone as a constraint is not a viable choice since noise is an undeniable physical constraint and may not be ignored while developing such algorithms.

As NCLMS algorithm is noise constrained only, a new constrained algorithm is established, by incorporating MAI variance as well as the noise variance, thus resulting in a generalized MAI plus noise constrained LMS (MNCLMS) adaptive algorithm. Since this proposed algorithm is generalized, this algorithm is able to deduce MAI constrained, noise constrained and zero constrained noise algorithms as special cases.

\section{ALGORITHM DEVELOPMENT}

In MIMO-CDMA system with $N$ transmitting and $M$ receiving antennas, output of $m^{\text {th }}$ matched-filter, matched to intended subscriber's (subscriber 1) spreading sequence consists of the desired user's component, $b_{m}^{l}$, MIMO-MAI, $U_{m}^{l}$ and the white Gaussian noise, $v_{m}^{l}$ as

$$
\begin{aligned}
y_{m}^{l} & =b_{m}^{l}+U_{m}^{l}+v_{m}^{l} \\
& =b_{m}^{l}+Z_{m}^{l}
\end{aligned}
$$

where $y_{m}^{l}$ is the output of the matched filter and $Z_{m}^{l}$ is the MIMO MAI and noise at the $m^{\text {th }}$ receiving antenna with variance $\sigma_{Z_{m}}^{2}$. The statistical characterization (variance) of MIMO CDMA MAI and noise is given in [7]. The MIMO CDMA MAI is given by

$$
\begin{aligned}
Z_{m}^{l} & =\sum_{n=1}^{N} \sum_{K=2}^{K} A^{K} b_{n}^{l, k} \rho^{K, 1} h_{m n}^{l}+v_{m}^{l} \\
& =U_{m}^{l}+v_{m}^{l}
\end{aligned}
$$

In $5, K$ is the number of subscribers, whereas $s_{n}^{l, k}(t)$ represents the rectangular signature waveform with a random signature sequence of $k^{\text {th }}$ subscriber defined as $(l-1) T_{b} \leq$ $t \leq l T_{b}$, whereas $T_{b}$ and $T_{c}$ represent bit period and the chip intervals respectively related by $N_{c}=T_{b} / T_{c}$. $b_{n}^{l, k}$ is the input bit stream of the $k^{\text {th }}$ subscriber, $h_{m n}^{l}$ is the $l^{\text {th }}$ channel tap between the $n^{\text {th }}$ transmitter and the $m^{\text {th }}$ receiver, $A^{k}$ is the transmitted amplitude of the $k^{\text {th }}$ subscriber and $v_{m}$ is the AWG noise having zero mean and variance $\sigma_{v}^{2}$ at the $m^{\text {th }}$ receiver. The cross-correlation between the signature sequences of subscriber $j$ and $k$ for the $l^{\text {th }}$ symbol is given as

$$
\begin{aligned}
\rho_{l}^{k, j} & =\int_{(l-1) T_{b}}^{l T_{b}} s_{n}^{k}(t) s_{n}^{j}(t) d t \\
& =\sum_{i=1}^{N_{c}} c_{l, i}^{k} c_{l, i}^{j}
\end{aligned}
$$

where $c_{l, i}^{k}$ is the normalized spreading sequence of the subscriber $k$ for the $l^{\text {th }}$ symbol.

The MIMO CDMA MAI consists of two random variables given as

$$
\begin{aligned}
U_{m}^{l} & =\sum_{n=1}^{N} \sum_{K=2}^{K} A^{K} b^{l, K} \rho^{K, 1} h_{m n}^{l} \\
& =\sum_{n=1}^{N} \mathscr{C} h_{m n}^{l},
\end{aligned}
$$

where $\mathscr{C}$ is MAI in AWGN environment [8]. The desired subscriber's component is written as

$$
\begin{gathered}
b_{m}^{l}=\left[\left(\mathbf{h}_{m 1}^{l}\right)^{T}\left(\mathbf{h}_{m 2}^{l}\right)^{T} \ldots\left(\mathbf{h}_{m n}^{l}\right)^{T}\right] \times \\
{\left[\left(\mathbf{x}_{1}^{l}\right)^{T}\left(\mathbf{x}_{2}^{l}\right)^{T} \ldots\left(\mathbf{x}_{N}^{l}\right)^{T}\right]^{T}}
\end{gathered}
$$

In (8), $\mathbf{h}_{m 1}^{l}=\left[h_{m 1}^{l} h_{m 1}^{l-1} \ldots h_{m 1}^{l-L+1}\right]^{T}$ is the time varying impulse response (TVIR) of the channels and is an $L \times 1$ vector. $\mathbf{x}_{n}^{l}=\left[x_{n}^{l} x_{n}^{l-1} \ldots x_{n}^{l-L+1}\right]^{T}$ is an $L \times 1$ vector. The filter impulse response (FIR) of the LE which consists of an FFF is given by

$$
\mathbf{w}_{n}^{l}=\left[\left(F_{n}^{l}\right)^{T}\right]
$$

In (9), $F_{n}^{l}$ is the $n^{\text {th }}$ multiple input, single output (MISO) FFF with dimension of $M L \times 1$, where $L$ is the taps of FFF and $M$ is the number of receivers.

The mean-squared error MSE) to be minimized is

$$
J\left(\mathbf{w}_{n}^{l}\right)=\left[e_{n}^{l}\right]^{2}
$$

where $e_{n}^{l}$ is the error between output of a matched filter and an adaptive filter and is shown to be

$$
e_{n}^{l}=\hat{x}_{n}-\mathbf{w}_{n}^{l} D_{n}^{l}
$$

here, $D_{n}^{l}$ is the combined input to the LE and is given by

$$
D_{n}^{l}=\left[\left(\mathbf{y}_{n}^{l}\right)^{T}\right]
$$

and is of the order of $(M L \times 1)$.

$$
\mathbf{y}_{n}^{l}=\left[\begin{array}{ll}
\left(\mathbf{y}_{1}^{l}\right)^{T}\left(\mathbf{y}_{2}^{l}\right)^{T} & \left(\mathbf{y}_{\mathrm{M}}^{l}\right)^{T}
\end{array}\right]^{T}
$$

is the input to the FFF of dimension $M L \times 1$ and is a collection of vectors consisting of $y_{m}^{l}$ given by

$$
\mathbf{y}_{m}^{l}=\left[\begin{array}{lll}
y_{m}^{l} y_{m}^{l-l} & y_{m}^{l-L+1}
\end{array}\right]^{T}
$$

and

$$
\begin{aligned}
\hat{\mathbf{x}}_{n} & =\mathbf{w}_{o}^{T} D_{n}^{l} \\
& =\mathbf{x}_{n}^{l}+\bar{v}_{n}^{l}
\end{aligned}
$$

or

$$
\mathbf{x}_{n}^{l}=\mathbf{w}_{o}^{T} D_{n}^{l}-\bar{v}_{n}^{l}
$$


$\bar{v}_{n}^{l}$ is the filtered noise which passes through FFF and is composed of MAI and noise.

Minimizing cost function in (10) over $\mathbf{w}_{n}^{l}$ will give an appropriate value at time $l$. In other words, $\mathbf{w}_{o p t}=H_{m n}^{l}$ (of size $1 \times N L$ matrix) with $\hat{J}(o p t)=\sigma_{\bar{v}_{n}^{l}}^{2}$ represents MSE. It is known that knowledge of $\sigma_{\bar{v}_{n}^{l}}^{2}$ [6] is helpful in selecting the search direction for an adaptive algorithm in multisubscriber case which is quite similar to the MNCLMS algorithm in single subscriber environment.

To minimize $J\left(\mathbf{w}_{n}^{l}\right)$ over $\mathbf{w}_{n}^{l}$ subject to the constraint $J\left(\mathbf{w}_{n}^{l}\right)=\sigma_{\bar{v}_{n}^{l}}^{2}$, the Lagrangian of this is given by

$$
J_{1}\left(\mathbf{w}_{n}^{l}, \lambda_{n}^{l}\right)=J\left(\mathbf{w}_{n}^{l}\right)+\lambda_{n}^{l}\left[J\left(\mathbf{w}_{n}^{l}\right)-\sigma_{\bar{v}_{n}^{l}}^{2}\right]
$$

Since critical values of $\lambda_{n}^{l}$ are not unique in this case so we are using an augmented Lagrangian to get rid of this issue by defining the under mentioned cost function

$$
J_{2}\left(\mathbf{w}_{n}^{l}, \lambda_{n}^{l}\right)=J\left(\mathbf{w}_{n}^{l}+\gamma \lambda_{n}^{l}\left[J\left(\mathbf{w}_{n}^{l}\right)-\sigma_{\bar{v}_{n}^{l}}^{2}\right]-\gamma_{n} \lambda_{n}^{2}\right.
$$

Solution to (18) is given by using the method used in [6] and is shown to be

$$
\mathbf{w}_{n}^{l+1}=\mathbf{w}_{n}^{l}+\mu_{n}^{l} e_{n}^{l} D_{n}^{l}
$$

where $\mu_{n}^{l}$ is the positive step size and is given by

$$
\begin{gathered}
\mu_{n}^{l}=\mu_{n}\left(1+\gamma_{n} \lambda_{n}^{l}\right), \\
m=1,2, \ldots, M \\
\lambda_{n}^{l+1}=\lambda_{n}+\beta_{n}\left[\frac{1}{2}\left(e_{n}^{l^{2}}-\sigma_{\bar{v}_{n}^{l}}^{2}\right)-\lambda_{n}^{l}\right], \\
m=1,2, \ldots, M
\end{gathered}
$$

Since channel taps are independent of spreading sequence as well as data sequence, the interferer's components are also independent of one another having zero mean.

The variance of MAI for an equal power can be written as

$$
U_{m}^{l}=A^{2}\left(\frac{K-1}{N_{C}}\right) \sum_{n=1}^{N} E\left[h_{m n}^{2}\right]
$$

whereas the variance of MAI in the unequal transmitted power is given by

$$
U_{m}^{l}=\frac{\sum_{k=2}^{k}\left(A^{k}\right)^{2}}{N_{C}} \sum_{n=1}^{N} E\left[h_{m n}^{2}\right]
$$

In (22) and (23), $E\left[h_{m n}^{2}\right]$ is the second moment of $E\left[h_{m n}\right]$.

\section{CONVERGENCE ANALYSIS}

In this section, convergence behavior of our proposed algorithm in the presence of MAI and noise for an LE receiver is analyzed. Following independent assumptions have been used while performing the convergence analysis [9].

Assumption 1: The input process $\left\{\mathbf{x}_{n}^{l}\right\}$ is an independent and identically distributed (i.i.d) random variable with auto correlation matrix of $\mathbf{R}_{x x}=E\left[\mathbf{x}_{n}^{T} \mathbf{x}_{n}\right]$.
Assumption 2: The noise sequence is a zero mean i.i.d. sequence, Gaussian random variable having variance $\sigma_{v_{n}}^{2}$. This sequence is independent of the input process.

Assumption 3: MAI in AWGN environment represented by $U_{m}^{l}$ is zero mean Gaussian random variable with variance $\sigma_{U_{m}}^{2}$. It is is independent of the input process as well as the noise.

Assumption 4: For any fixed time. say $l$, step size $\mu_{n}^{l}$ and weight vector $\mathbf{v}_{n}^{l}$ (defined later) are statistically independent of each another.

The above mentioned assumptions are justified as follows

Although assumption 1 is not true in reality but is commonly used in literature and it has been shown in the literature that simulation results under this condition closely match with the analytical results.

Assumption 2 termed as independent assumption, is also commonly used in the literature and could be justified in many cases.

Gaussian appropriation of MAI in AWGN is extensively used in the adaptive filtering literature [10]. Since MAI is independent of the noise process, assumption 3 can be validated. In this way, MAI and noise is independent of the input process as well.

Generally $\mu_{n}^{l}$ and $\mathbf{v}_{n}^{l}$ are dependent but if the parameters are chosen in such a way that steady-state variance of $\mu_{n}^{l}$ and or $\mathbf{v}_{n}^{l}$ is small, assumption 4 can be justified.

$\mathbf{x}_{k}$ and $\mathbf{x}_{n}$ are also independent as both are uncorrelated for $n \neq k$. Thus the input vector $\mathbf{x}_{n}$ and the weight error vector (defined later) $\mathbf{v}_{n}^{l}$ are also independent [11].

Weight update equation of the proposed algorithm is written as

$$
\mathbf{w}_{n}^{l+1}=\mathbf{w}_{n}^{l}+\mu_{n}^{l} e_{n}^{l} D_{n}^{l}
$$

Where $n=1,2 \ldots \ldots ., N$ represents the $n^{\text {th }}$ stream.

If $\mathbf{w}_{n(o p t)}$ is said to be the optimum value of weight according to the Wiener solution then weight error vector $\mathbf{v}_{n}^{l}$ can be defined as

$$
\mathbf{v}_{n}^{l}=\mathbf{w}_{n(o p t)}-\mathbf{w}_{n}^{l}
$$

Subtracting $\mathbf{w}_{n(\text { opt }}$ from both sides of (19) results in

$$
\begin{gathered}
\mathbf{v}_{n}^{l+1}+\mathbf{w}_{n(o p t)}=-\mathbf{w}_{n}^{l}+\mathbf{w}_{n(o p t)}-\mu_{n}^{l} e_{n}^{l} D_{n}^{l} \\
\mathbf{v}_{n}^{l+1}=\mathbf{v}_{n}^{l}-\mu_{n}^{l} e_{n}^{l} D_{n}^{l}
\end{gathered}
$$

The desired response of the decision device $\left(\tilde{\mathbf{x}}_{n}^{l}\right)$ can be expressed as

$$
\tilde{\mathbf{x}}_{n}^{l}=\mathbf{w}_{(\text {opt })}^{T} D_{n}^{l}
$$

By using 27, it can be shown in case of system identification scenario, $e_{n}^{l}$ could be setup as below:

$$
e_{n}^{l}=\mathbf{w}_{(o p t)}^{T}-\left(\mathbf{w}_{n}^{l}\right)^{T}
$$

For $n=1,2, \ldots N$.

(28) can be compactly written as

$$
\begin{aligned}
e_{n}^{l} & =\left(\mathbf{v}_{n}^{l}\right)^{T} D_{n}^{l} \\
& =\left(D_{n}^{l}\right)^{T} \mathbf{v}_{n}^{l}
\end{aligned}
$$


Now the recursion of weight error vector can be shown as

$$
\begin{aligned}
& \mathbf{v}_{n}^{l+1}=\mathbf{v}_{n}^{l}-\mu_{n}^{l} D_{n}^{l}\left(D_{n}^{l}\right)^{T} \mathbf{v}_{n}^{l} \\
& \mathbf{v}_{n}^{l+1}=\left\{\mathbf{I}-\mu_{n}^{l} D_{n}^{l}\left(D_{n}^{l}\right)^{T}\right\} \mathbf{v}_{n}^{l}
\end{aligned}
$$

Taking expectation on both sides of (31) with the assumptions made earlier, will yield

$$
\overline{\mathbf{v}}_{n}^{l}=\left[\mathbf{I}-\bar{\mu}_{n}^{l} E\left\{D_{n}^{l}\left(D_{n}^{l}\right)^{T}\right\}\right] \overline{\mathbf{v}}_{n}^{l}
$$

$\bar{\mu}_{n}^{l}=E\left[\mu_{n}^{l}\right]$ is the mean step size and $\overline{\mathbf{v}}_{n}^{l}=E\left[\mathbf{v}_{n}^{l}\right]$ is the weight error vector in the equation above. Whereas

or

$$
E\left[D_{n}^{l}\left(D_{n}^{l}\right)^{T}\right]=E\left[\left(\mathbf{y}_{n}^{l}\right)^{T}\right]\left[\left(\mathbf{y}_{n}^{l}\right)^{T}\right]^{T}
$$

$$
\tilde{R}=E\left[\left(\mathbf{y}_{n}^{l}\right)^{T}\right]^{T}\left[\left(\mathbf{y}_{n}^{l}\right)^{T}\right]
$$

or

$$
\tilde{R}=E\left[D_{n}^{l}\left(D_{n}^{l}\right)^{T}\right]
$$

where $\tilde{R}$ is the correlation matrix of input process

A. Auto-correlation Structure of MIMO-CDMA Linear Equalizer (LE)

The correlation matrix $\tilde{R}$ is given by

$$
\tilde{R}=R_{D_{n}^{l} D_{n}^{l}}
$$

\section{B. Eigenvalues of Linear Equalizer (LE)}

If $\Upsilon_{1}, \Upsilon_{2}, \Upsilon_{3} \ldots \Upsilon_{N}$ represent the eigenvalues of $\tilde{R}$, then the an important condition for the convergence in the mean is given as

$$
\left|1-\bar{\mu}_{n}^{l} \Upsilon_{N}\right|<1,
$$

The value of $\bar{\mu}_{n}^{l}$ is bounded in the range

$$
\begin{aligned}
0 & <\bar{\mu}_{n}^{l} \\
& <\frac{2}{\Upsilon_{\max }}
\end{aligned}
$$

where $Y_{\max }$ is the maximum eigenvalue of $\tilde{R}$. By using a strong and a much simpler condition for convergence of mean weight error vector [12] can be given as

$$
\mu_{\max }<\frac{2}{\Upsilon_{\max }}
$$

\section{Transient Analysis of the Proposed Algorithm}

Transient analysis of an adaptive algorithm is very important to observe the convergence behavior of an adaptive algorithm and to derive steady-state expressions for different error performance measures, such as EMSE and mean-square deviation (MSD). Basically, transient analysis is the observation of the time-evolution of the adaptive algorithms when there are variations in the signal statistics; or in other words to study the learning mechanism of an adaptive algorithm. Energy conservation method is used to carry out the transient analysis [9].

\section{A. Error Measures}

Transient analysis of an adaptive algorithm deals with the time evaluation of of $E\left[\left|e_{n}^{l}\right|^{2}\right]$ and $E\left[\left\|\mathbf{v}_{n}^{l}\right\|^{2}\right]$, where $\mathbf{v}_{n}^{l}$ is the weight error vector and is given by

$$
\mathbf{v}_{n}^{l}=\mathbf{w}_{n(o p t)}^{l}-\mathbf{w}_{n}^{l}
$$

For some symmetric positive definite weighting matrix $\Omega$,We define, weighted a priori and a posteriori estimation errors as

$$
e_{a n}^{\Omega}=\left(\mathbf{v}_{n}^{l}\right)^{T} \Omega D_{n}^{l}
$$

and

$$
e_{p n}^{\Omega}=\left(\mathbf{v}_{n}^{l+1}\right)^{T} \Omega D_{n}^{l}
$$

For the case when, $\Omega=I$, the weighted a priori and a posteriori estimation errors defined above will be reduced to a standard a priori and a posteriori estimation errors, respectively, i.e

$$
e_{a n}^{l}=\left(D_{n}^{l}\right)^{T} \mathbf{v}_{n}^{l}
$$

and

$$
e_{p n}^{l}=\left(D_{n}^{l}\right)^{T} \mathbf{v}_{n}^{l+1}
$$

It will be elaborated later in that different choices of $\Omega$ will yield different performance measures for the evaluation of an adaptive filter.

Since,

$$
\begin{gathered}
e_{n}^{l}=\mathbf{x}_{n}^{l}-\left(\mathbf{w}_{n}^{l}\right)^{T} D_{n}^{l} \\
=\left(\mathbf{w}_{(o p t)}\right)^{T}-\bar{v}_{n}^{l}-\left(\mathbf{w}_{n}^{l}\right)^{T} D_{n}^{l} \\
e_{n}^{l}=\left(\mathbf{v}_{n}^{l}\right)^{T} D_{n}^{l}-v_{n}^{l} \\
=e_{a n}^{l}-\bar{v}_{n}^{l}
\end{gathered}
$$

Also, by using (40) and and (26) it can be shown that

$$
e_{p n}^{\Omega, l}=e_{a n}^{\Omega, l}-\mu_{n}^{l}\left\|D_{n}^{l}\right\|_{\Omega}^{2} e_{n}^{l}
$$

\section{B. Performance Measures}

The EMSE of the proposed algorithm is given by

$$
E M S E=E\left[\left|e_{a n}^{l}\right|^{2}\right]
$$

and steady state EMSE is

$$
E\left[\left|e_{a n}^{l}\right|^{2}\right]=\xi_{\infty}
$$




\section{Fundamental Weighted Energy Relation}

The fundamental weighted-energy conservation relation [9] is used in this section to develop the framework for the transient analysis of the proposed MNCLMS algorithm. (44) can be expressed as

$$
e_{n}^{\Omega, l}=\frac{e_{n}^{\Omega, n}-e_{p n}^{\Omega, l}}{\mu_{n}^{l}\left\|D_{n}^{l}\right\|_{\Omega}^{2}}
$$

By using (26) and (47) ,the following equation can be established

$$
\overline{\mathbf{v}}_{n}^{l+1}=\mathbf{v}_{n}^{l}-\frac{D_{n}^{l}}{\left\|D_{n}^{l}\right\|_{\Omega}^{2}}\left[e_{a n}^{\Omega, l}-e_{n}^{\Omega, l}\right]
$$

The fundamental weighted-energy conservation relation can be shown as

$$
\mathbf{v}_{n}^{l+1}+\frac{1}{\left\|D_{n}^{l}\right\|_{\Omega}^{2}}\left|e_{a n}^{\Omega}\right|^{2}=\left\|\mathbf{v}_{n}^{l}\right\|_{\Omega}^{2}+\frac{1}{\left\|D_{n}^{l}\right\|_{\Omega}^{2}}\left|e_{p n}^{l, \Omega}\right|^{2}
$$

49 describes, how the weighted energies of the error quantities evolve with time. Different choices of $\Omega$ will yield different performance measures for the evaluation of an adaptive filter[9].

\section{Time Evolution of the Weighted Variance}

This section deals with derivation of time evolution of the weighted variance for the proposed MNCLMS algorithm using the fundamental weighted-energy conservation relation equation (49). Substituting (44) into (49) and taking expectation on both sides will result in

$$
\begin{aligned}
E\left[\left\|\mathbf{v}_{n}^{l+1}\right\|_{\Omega}^{2}\right]= & E\left[\left\|\mathbf{v}_{n}^{l}\right\|_{\Omega}^{2}\right]- \\
& 2 \overline{\mu_{n}^{l} E}\left[e_{a n}^{l, \Omega} e_{n}\right]+\overline{\left(\mu_{n}^{l}\right)^{2}} E\left[\left\|D_{n}^{l}\right\|_{\Omega}^{2} e_{n}^{2}\right]
\end{aligned}
$$

where $\overline{\left(\mu_{n}^{l}\right)^{2}}$ is $E\left[\left(\mu_{n}^{l}\right)^{2}\right]$. Next, the expectations in the second and third terms on the right hand side of equation (50) is evaluated by using the following assumption

A5): For any constant matrix $\Omega$ and for all $l, e_{a n}^{l}$ and $e_{a n}^{\Omega}$ are jointly Gaussian.

This assumption seems reasonable for longer filters using the central limit theorem [13], [14], [4]. So, $E\left[e_{a n}^{l, \Omega} e_{n}\right]$ can be simplified as

$$
\begin{aligned}
& E\left[e_{a n}^{l, \Omega} e_{n}\right]=E\left[\left(D_{n}^{l}\right)^{T} \Omega \mathbf{v}_{n}^{l}\left(D_{n}^{l}\right)^{T} I \mathbf{v}_{n}^{l}\right]= \\
& E\left[\left(\mathbf{v}_{n}^{l}\right)^{T}\left(\Omega\left(D_{n}^{l}\right)\left(D_{n}^{l}\right)^{T} I\right) \mathbf{v}_{n}^{l}\right] \\
& =E\left[\left\|\mathbf{v}_{n}^{l}\right\|_{\Omega E\left[\left(D_{n}^{l}\right)\left(D_{n}^{l}\right)^{T}\right]}^{2}\right]=E\left[\left\|\mathbf{v}_{n}^{l}\right\|_{\Omega \tilde{R}}^{2}\right]
\end{aligned}
$$

Now, $E\left[\left\|D_{n}^{l}\right\|_{\Omega}^{2} e_{n}^{2}\right]$ is being solved by using the following assumption

A6): The adaptive filter is long enough so that $\left\|D_{n}^{l}\right\|_{\Omega}^{2}$ and $e_{n}^{2}$ are uncorrelated [9].
This assumption is more realistic when the filter length gets longer[9]. As MAI plus noise is independent of $D_{n}^{l}$, expectation $E\left[\left\|D_{n}^{l}\right\|_{\Omega}^{2} e_{n}^{2}\right]$ can be simplified as

$$
\begin{aligned}
E\left[\left\|D_{n}^{l}\right\|_{\Omega}^{2} e_{n}^{2}\right]=E\left[\left\|D_{n}^{l}\right\|_{\Omega}^{2}\right] E\left[e_{n}^{2}\right]= \\
E\left[\left\|D_{n}^{l}\right\|^{2}\right]\left(E\left[\left(e_{a n}^{l}\right)^{2}\right]-\sigma_{\bar{v}_{n}^{l}}^{2}\right)
\end{aligned}
$$

Now using (51) and (52) and, (50) can be written as

$$
\begin{aligned}
E\left[\left\|\mathbf{v}_{n}^{l+1}\right\|_{\Omega}^{2}\right]=E\left[\left\|\mathbf{v}_{n}^{l}\right\|_{\Omega}^{2}\right]-2 \bar{\mu}_{n}^{l} E\left[\left\|\mathbf{v}_{n}^{l}\right\|_{\Omega \tilde{R}}^{2}\right]+ & \\
& \overline{\left(\mu_{n}^{l}\right)^{2}} E\left[\left\|D_{n}^{l}\right\|_{\Omega}^{2}\right]\left(\xi_{n}^{l}-\sigma_{\bar{v}_{n}^{l}}^{2}\right)
\end{aligned}
$$

(53) shows the time evaluation or the transient behavior of the weighted variance $E\left[\left\|\mathbf{v}_{n}^{l}\right\|_{\Omega}^{2}\right]$ for any constant weight matrix $\Omega$. Different performance measures can be achieved by the proper choice of the weight matrix $\Omega$.

\section{E. Constructing the Learning Curves for the Excess Mean Square Error (EMSE)}

Learning curves for the EMSE can be constructed by using $E M S E=E\left[\left(e_{a n}^{l}\right)^{2}\right]=E\left[\left\|\mathbf{v}_{n}^{l}\right\|_{\tilde{R}}^{2}\right]$. If $\Omega=I, \tilde{R}, \ldots, \tilde{R}^{N-1}$, a set of relations can be obtained. Now by using the CayleyHamilton theorem, following can be established

$$
\Omega=-p_{0} I-p_{1} \tilde{R}-\ldots-p_{N-1} \tilde{R}^{N-1}
$$

where

$p(x) \triangleq \operatorname{det}(x I-\tilde{R})=p_{0}+p_{1} x+\ldots+p_{N-1} x^{N-1}+x^{N}$

is the characteristic polynomial of $\tilde{R}$.Consequently,

$$
\begin{aligned}
& E\left[\left\|\mathbf{v}_{n}^{l+1}\right\|_{\tilde{R}^{N-1}}^{2}\right]=E\left[\left\|\mathbf{v}_{n}^{l}\right\|_{\tilde{R}^{N-1}}^{2}\right]+2 \overline{\mu_{n}^{l}} \\
& \left(p_{0} E\left[\left\|\mathbf{v}_{n}^{l}\right\|_{I}^{2}\right]+p_{1} E\left[\left\|\mathbf{v}_{n}^{l}\right\|_{\tilde{R}}^{2}\right]+\ldots\right. \\
& \left.+p_{N-1} E\left[\left\|\mathbf{v}_{n}^{l}\right\|_{\tilde{R}^{N-1}}^{2}\right]\right)
\end{aligned}
$$

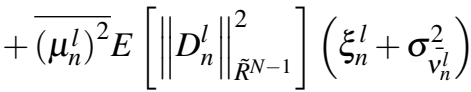

So,

$\Upsilon_{n}^{l}$

$=\left[\begin{array}{cccccc}1 & -2 \overline{\mu_{n}^{l}} & 0 & \cdots & 0 & 0 \\ 0 & 1 & -2 \overline{\mu_{n}^{l}} & 0 & \cdots & 0 \\ \vdots & 0 & 1 & -2 \overline{\mu_{n}^{l}} & \ldots & \vdots \\ 0 & 0 & 0 & 1 & -2 \overline{\mu_{n}^{l}} & 0 \\ 0 & 0 & \cdots & 0 & \overline{1} & -2 \overline{\mu_{n}^{l}} \\ 2 \overline{\mu_{n}^{l}} p_{0} & 2 \bar{\mu}_{n}^{l} p_{1} & 2 \overline{\mu_{n}^{l}} p_{2} & \cdots & 2 \overline{\mu_{n}^{l}} p_{N-2} & 1+2 \bar{\mu}_{n}^{l} p_{N-1}\end{array}\right]$

And

$$
\varpi_{n}^{l}=\left[\begin{array}{c}
E\left[\left\|\mathbf{v}_{n}^{l}\right\|_{I}^{2}\right] \\
E\left[\left\|\mathbf{v}_{n}^{l}\right\|_{\tilde{R}}^{2}\right] \\
\vdots \\
E\left[\left\|\mathbf{v}_{n}^{l}\right\|_{\tilde{R}_{N-1}}^{2}\right]
\end{array}\right]
$$


Similarity

$$
\mathrm{a}_{n}^{l}=\left[\begin{array}{c}
E\left[\left\|D_{n}^{l}\right\|_{I}^{2}\right. \\
E\left[\left\|D_{n}^{l}\right\|_{\tilde{R}}^{2}\right] \\
\vdots \\
E\left[\left\|D_{n}^{l}\right\|^{2}\right]
\end{array}\right]
$$

By combining 57,58 and 59 ,

$$
\varpi_{n}^{l+1}=\Upsilon_{n}^{l} \varpi_{n}^{l}+\overline{\left(\mu_{n}^{l}\right)^{2}}\left(\xi_{n}^{l}+\sigma_{\bar{v}_{n}^{l}}^{2}\right) \mathrm{a}_{n}^{l}
$$

\section{Steady-State AnAlysis of The MNCLMS}

\section{ALGORITHMS}

Steady-state analysis of an adaptive filter is performed to study the behavior of steady-state EMSE and MSD. Steadystate performance measures is also obtained by analyzing (50) when $l \rightarrow \infty$ which is presented in the next section i.e

$$
\lim _{l \rightarrow \infty} E\left[\left\|\mathbf{v}_{n}^{l+1}\right\|_{\Omega}^{2}\right]=\lim _{l \rightarrow \infty} E\left[\left\|\mathbf{v}_{n}^{l}\right\|^{2}\right]
$$

So at steady-state (53) will become

$$
2 \bar{\mu}_{n}^{\infty} \lim _{l \rightarrow \infty} E\left[\left\|\mathbf{v}_{n}^{l}\right\|_{\Omega \tilde{R}}^{2}\right]=\overline{\left(\mu_{n}^{\infty}\right)^{2}} \lim _{l \rightarrow \infty} E\left[\left\|D_{n}^{l}\right\|_{\Omega}^{2}\right]\left(\xi_{n}^{\infty}+\sigma_{\bar{v}_{n}^{l}}^{2}\right)
$$

Where $\bar{\mu}_{n}^{\infty}, \overline{\left(\mu_{n}^{\infty}\right)^{2}}$ and $\xi_{n}^{\infty}$ are steady-state values of $\bar{\mu}_{n}^{l}$, $\overline{\left(\mu_{n}^{l}\right)^{2}}$ and $\xi_{n}^{l}$ respectively. Now using (20) and (21) it can be shown that

$$
\begin{array}{r}
\left(\bar{\mu}_{n}^{l}\right)^{2}=\overline{\left(\mu_{n}^{l}\right)^{2}}\left(2 \gamma \lambda_{n}^{l}+\gamma^{2}\left(\bar{\lambda}_{n}^{l}\right)^{2}\right) \\
\left(\lambda_{n}^{l+1}\right)^{2}=(1-\beta)^{2}\left(\lambda_{n}^{l}\right)^{2}+\beta(1-\beta) \bar{\lambda}_{n}^{l} \xi_{n}^{l}+ \\
\frac{\beta^{2}}{4}\left[E\left[\left|e_{n}^{l}\right|^{4}\right]\right. \\
\left.-2 \sigma_{\bar{v}_{n}^{l}}^{2}\left(\xi_{n}^{l}+\sigma_{\bar{v}_{n}^{l}}^{2}\right)+\sigma_{\bar{v}_{n}^{l}}^{4}\right]
\end{array}
$$

(64) can be written compactly as

$\left(\lambda_{n}^{l+1}\right)^{2}=(1+\beta)^{2} \overline{\left(\lambda_{n}^{l}\right)^{2}}+\beta(1-\beta) \bar{\lambda}_{n}^{l} \xi_{n}^{l}+\frac{\beta}{2} \xi_{n}^{l} \sigma_{\overline{v_{n}^{l}}}^{2}$

If we define mean Lagrangian multiplier as $\bar{\lambda}_{n}^{l}=E\left[\lambda_{n}^{l}\right]$, it can be shown that at steady-state

$$
\bar{\lambda}_{n}^{l}=\frac{\xi_{n}^{\infty}}{2}
$$

Similarly, if $\bar{\mu}_{n}^{l}=E\left[\mu_{n}^{l}\right]$, it can be shown that

$$
\bar{\mu}_{n}^{\infty}=\mu\left(1+\gamma \frac{\xi_{n}^{\infty}}{2}\right)
$$

(63) can be expressed as

$$
\overline{\left(\mu_{n}^{\infty}\right)^{2}}=\mu^{2}\left[1+2 \gamma \lambda_{n}^{\infty}+\gamma^{2}\left(\lambda_{n}^{\infty}\right)^{2}\right]
$$

(65) is written as

$$
\overline{\left(\lambda_{n}^{\infty}\right)^{2}}=\frac{1}{(2-\beta)}\left[\frac{(1-\beta)}{2}\left(\xi_{n}^{\infty}\right)^{2}+\beta \xi_{n}^{\infty} \sigma_{\bar{v}_{n}^{l}}^{2}\right]
$$

Using (62) with $\Omega=I$,

$$
\begin{aligned}
& 2 \xi_{n}^{\infty}+\gamma\left(\xi_{n}^{\infty}\right)^{2}= \mu \operatorname{Tr}(\tilde{R}) \xi_{n}^{\infty}+\mu \operatorname{Tr}(\tilde{R}) \sigma_{\bar{v}_{n}^{l}}^{2}+ \\
& \mu \gamma \operatorname{Tr}(\tilde{R})\left(\xi_{n}^{\infty}\right)^{2}+ \\
& \mu \gamma \operatorname{Tr}(\tilde{R}) \sigma_{v_{n}^{l}}^{2} \xi_{n}^{\infty} \\
&+\mu \gamma^{2}\left(\frac{1}{2-\beta}\right)\left(\frac{1-\beta}{2}\right) \operatorname{Tr}(\tilde{R})\left(\xi_{n}^{\infty}\right)^{3} \\
&+\mu \gamma^{2}\left(\frac{1}{2-\beta}\right)\left(\frac{1-\beta}{2}\right) \operatorname{Tr}(\tilde{R}) \\
&+\mu \beta \operatorname{Tr}(\tilde{R}) \sigma_{\bar{v}_{n}^{l}}^{2}\left(\xi_{n}^{\infty}\right)^{2}+ \\
& \mu \beta \operatorname{Tr}(\tilde{R}) \sigma_{\bar{v}_{n}^{l}}^{2} \xi_{n}^{\infty}
\end{aligned}
$$

(70) is cubic in terms of $\xi_{n}^{\infty}$ and can be expressed as

$$
A\left(\xi_{n}^{\infty}\right)^{3}+B\left(\xi_{n}^{\infty}\right)^{2}+C \xi_{n}^{\infty}+D=0
$$

In 71above,

$$
A=-\mu \gamma^{2} \operatorname{Tr}(\tilde{R})\left(\frac{1}{2-\beta}\right)\left(\frac{1-\beta}{2}\right)
$$

$B=\gamma-\mu \operatorname{Tr}(\tilde{R})\left[\gamma+\sigma_{\overline{v_{n}^{l}}}^{2}\left\{\left(\frac{1}{1-\beta}\right)\left(\frac{1-\beta}{2}\right)+\beta\right\}\right]$

$$
C=2-\mu \operatorname{Tr}(\tilde{R})\left[1+\left(\gamma+\beta \sigma_{\overline{v_{n}^{l}}}^{2}\right) \sigma_{\bar{v}_{n}^{l}}^{2}\right]
$$

And

$$
D=-\mu \operatorname{Tr}(\tilde{R}) \sigma_{v_{n}^{l}}^{2}
$$

Now assuming that at steady-state, $\mu \operatorname{Tr}(\tilde{R}) \ll 1$ [15], it can be shown that at steady-state, the value of $\xi_{n}^{\infty}$ is close to zero meaning that higher powers of $\xi_{n}^{\infty}$ can be ignored. Consequently, (71) will become,

$$
C \xi_{n}^{\infty}+D=0
$$

or

$$
\xi_{n}^{\infty}=\frac{-D}{C}
$$

Hence, steady-state EMSE of the proposed MNCLMS algorithm in the presence of MAI and noise can be shown to be

$$
\xi_{n(M N C L M S)}^{\infty} \approx \frac{\mu \operatorname{Tr}(\tilde{R}) \sigma_{\bar{v}_{n}^{l}}^{2}}{2-\mu \operatorname{Tr}(\tilde{R})\left[1+\left(\gamma+\beta \sigma_{\overline{v_{n}^{l}}}^{2}\right) \sigma_{\bar{v}_{n}^{l}}^{2}\right]}
$$

By using the same procedure developed above, steadystate EMSE of the LMS, NCLMS, ZNCLMS, and MCLMS algorithms in the presence of MAI and noise can be shown, respectively to be

$$
\xi_{n(L M S)}^{\infty} \approx \frac{\mu \operatorname{Tr}(\tilde{R}) \sigma_{\overline{v_{n}}}^{2}}{2}
$$

$\xi_{N C L M S}^{\infty} \approx \frac{-\left(2+\gamma \sigma_{U}^{2}\right)+2 \mu \operatorname{Tr}(\tilde{R}) \sigma_{U}^{2}\left[1+\gamma \sigma_{U}^{4}+\gamma^{2}\left(\frac{1-\beta}{2-\beta}\right)\left\{\sigma_{U}^{2}+\sigma_{U}^{4}\right\}\right]}{\left(2+\gamma \sigma_{U}^{2}\right)-2 \mu \operatorname{Tr}(\tilde{R})}$ 


$$
\begin{aligned}
& \xi_{\text {ZNCLMS }}^{\infty} \\
& \approx \frac{-\left(2+\gamma \sigma_{\bar{v}_{n}^{l}}^{2}\right)+2 \mu \operatorname{Tr}(\tilde{R}) \sigma_{\tilde{v}_{n}^{l}}^{2}\left[1+\gamma \sigma_{\bar{v}_{n}^{l}}^{4}+\gamma^{2}\left(\frac{1-\beta}{2-\beta}\right)\left\{\sigma_{\bar{v}_{n}^{l}}^{2}+\sigma_{\bar{v}_{n}^{l}}^{4}\right\}\right]}{\left(2+\gamma \sigma_{\bar{v}_{n}^{l}}^{2}\right)-2 \mu \operatorname{Tr}(\tilde{R})}
\end{aligned}
$$

$$
\approx \frac{-\left(2+\gamma \sigma_{\bar{v}_{n}^{l}}^{2}\right)+2 \mu \operatorname{Tr}(\tilde{R}) \sigma_{\bar{v}_{n}^{l}}^{2}\left[1+\gamma \sigma_{\bar{v}_{n}^{l}}^{4}+\gamma^{2}\left(\frac{1-\beta}{2-\beta}\right)\left\{\sigma_{\bar{v}_{n}^{l}}^{2}+\sigma_{\bar{v}_{n}^{l}}^{4}\right\}\right]}{\left(2+\gamma \sigma_{\bar{v}_{n}^{l}}^{2}\right)-2 \mu \operatorname{Tr}(\tilde{R})}
$$

\section{TRACKING ANALYSIS OF THE MNCLMS \\ AlgorithmS FOR THE RANDOM WALK CHANNEL IN THE PRESENCE OF MAI AND NOISE}

Tracking analysis of an adaptive filter is performed to study its ability to track down the time variations in the channel. This is due to the fact that statistical properties of the weight vector and error signals are able to track the changes in the input signal variation by relying on instantaneous data [9]. In this section, tracking analysis of the proposed algorithm is performed for a random walk model.

\section{A. Random Walk Model}

This section deals with the tracking analysis of the MNCLMS algorithm performed for a random walk channel. A general framework for the tracking analysis of adaptive algorithms is used in this section which can handle random system nonstationarities [16]. This framework is based on an energy conservation relation and is valid for adaptive algorithms whose recursion is of the form

$$
\mathbf{w}_{n}^{l+1}=\mathbf{w}_{n}^{l}+\mu_{n}^{l} D_{n}^{l} f\left(e_{n}^{l}\right)
$$

Where $f\left(e_{n}^{l}\right)$ represents a general scalar function of the output estimation error $e_{n}^{l}$. For an LMS algorithm $f\left(e_{n}^{l}\right)=e_{n}^{l}$.

The random walk model for a channel is given by

$$
\mathbf{w}_{n}^{l+1}=\mathbf{w}_{o, n}^{l}+q_{n}^{l}
$$

$q_{n}^{l}$ in (84) is assumed to be zero mean, i.i.d, with a positive definite co-variance matrix $E\left[q_{n} q_{n}^{T}\right]=Q$ and is also statistically independent of the input regressor and the MAI plus noise, whereas $\mathbf{w}_{n}^{o}$ is the unknown system to be tracked. For tracking analysis of an adaptive algorithm, a very important measure is its steady-state tracking $\operatorname{EMSE}\left(\xi_{n}^{\infty}\right)$ defined as

$$
\begin{aligned}
\xi_{n}^{\infty} & =\lim _{l \rightarrow \infty} E\left[\left|e_{a n}^{l}\right|^{2}\right] \\
& =E\left[\left\|\mathbf{v}_{n}^{l}\right\|_{\tilde{R}}^{2}\right]
\end{aligned}
$$

Where $\mathbf{v}_{n}^{l}=\mathbf{w}_{n}^{o}-\mathbf{w}_{n}^{l}$ is the weight error vector for the random walk channel.

\section{B. Fundamental Energy Relation for the Random Walk Chan-} nel

In this section, the fundamental energy conservation relation [9] is used to develop the framework for the tracking analysis of the proposed MNCLMS algorithm. By using (19) and (84), it can be shown that

$$
\mathbf{v}_{n}^{l+1}=\mathbf{v}_{n}^{l}-\mu_{n}^{l} e_{n}^{l} D_{n}^{l}+q
$$

Consider the (83), which is given by

$$
\mathbf{w}_{n}^{l+1}=\mathbf{w}_{n}^{l}+\mu_{n}^{l} D_{n}^{l} f\left(e_{n}^{l}\right)
$$

Subtracting both sides of the (87) from $\mathbf{w}_{0, n}^{l+1}$

$$
\left(\mathbf{w}_{o, n}^{l+1}-\mathbf{w}_{n}^{l+1}\right)=\left(\mathbf{w}_{o, n}^{l+1}-\mathbf{w}_{n}^{l}\right)-\mu_{n}^{l} D_{n}^{l} f\left(e_{n}^{l}\right)
$$

In case of an LMS algorithm, $f\left(e_{n}^{l}\right)=e_{n}^{l}$, so (88) becomes

$$
\mathbf{w}_{o, n}^{l+1}-\mathbf{w}_{n}^{l+1}=\left(\mathbf{w}_{o, n}^{l+1}-\mathbf{w}_{n}^{l}\right)-\mu_{n}^{l} D_{n}^{l} e_{n}^{l}
$$

Now we transpose both sides of (89)

$$
\left(\mathbf{w}_{o, n}^{l+1}-\mathbf{w}_{n}^{l+1}\right)^{T}=\left(\mathbf{w}_{o, n}^{l+1}-\mathbf{w}_{n}^{l}\right)^{T}-\mu_{n}^{l} e_{n}^{l}\left(D_{n}^{l}\right)^{T}
$$

By multiplying both sides of (90) with $D_{n}^{l}$ from left yields

$$
\left(\mathbf{w}_{o, n}^{l+1}-\mathbf{w}_{n}^{l+1}\right)^{T} D_{n}^{l}=\left(\mathbf{w}_{o, n}^{l+1}-\mathbf{w}_{n}^{l}\right)^{T} D_{n}^{l}-\mu_{n}^{l} e_{n}^{l}\left(D_{n}^{l}\right)^{T} D_{n}^{l}
$$

Equation (91) in terms of a priori error and a posteriori errors can be expressed as

$$
e_{p, n}^{l}=e_{a, n}^{l}-\mu_{n}^{l} e_{n}^{l}\left\|D_{n}^{l}\right\|^{2}
$$

For the case when $\Omega=I, 86$ becomes

$$
\mathbf{v}_{n}^{l+1}=\mathbf{v}_{n}^{l}-D_{n}^{l}\left[\frac{e_{a n}^{l}-e_{p n}^{l}}{\left\|D_{n}^{l}\right\|^{2}}\right]+q_{n}^{l}
$$

By evaluating the energies on both sides of (93) We get

$$
\left\|\mathbf{v}_{n}^{l+1}-q_{n}^{l}\right\|^{2}+\frac{1}{\left\|D_{n}^{l}\right\|^{2}}\left|e_{a n}^{l}\right|^{2}=\left\|\mathbf{v}_{n}^{l l}\right\|^{2}+\frac{1}{\left\|D_{n}^{l}\right\|^{2}}\left|e_{p n}^{l}\right|^{2}
$$

Since $q_{n}^{l}$ is a zero mean stationary random vector and is independent of the input regressor vector and the MAI plus noise, so (94), can be expressed as

$$
\left\|\mathbf{v}_{n}^{l+1}\right\|^{2}-\left\|q_{n}^{l}\right\|^{2}+\frac{1}{\left\|D_{n}^{l}\right\|^{2}}\left|e_{a n}^{l}\right|^{2}=\left\|\mathbf{v}_{n}^{l}\right\|^{2}+\frac{1}{\left\|D_{n}^{l}\right\|^{2}}\left|e_{p n}^{l}\right|^{2}
$$

(95) is the random walk tracking model for MNCLMS algorithm. The energy relation is used to evaluate the excess mean-square error at steady state. 
TABLE I

COMPUTATIONAL COMPLEXITY PER ITERATION FOR DIFFERENT ALGORITHMS FOR REAL VALUED DATA IN TERMS OF THE REAL MULTIPLICATIONS, REAL ADDITIONS AND REAL DIVISIONS

\begin{tabular}{|c|c|c|}
\hline Algorithm & $\times$ & + \\
\hline \hline LMS & $2 L+1$ & $L$ \\
\hline RLS & $L^{2}+5 L+1$ & $L^{2}+3 L$ \\
\hline MNCLMS[4] & $2 L+1$ & $2 L+6$ \\
\hline MIMO-MNCLMS(LE) & $2 M L+8$ & $2 M L+4$ \\
\hline
\end{tabular}

\section{Tracking Steady-State EMSE of the MNCLMS Algorithms}

A7): $q_{n}$ is a zero-mean stationary random vector process with a positive definite covariance matrix $Q$ and is statistically independent of the input regressor vector $\mathbf{x}_{n}^{l}$ and MAI plus noise $Z_{m}^{l}$ sequence .

Taking expectation on both sides of equation (95) and using equation (44) assumption A7) and the fact that at steady-state, $\mathbf{v}_{n}^{l+1}=\mathbf{v}_{n}^{l}$, the following equation can be obtained

$$
2 E\left[\mu_{n}^{\infty} \xi_{n}^{\infty}\right]=\operatorname{Tr}\left(Q_{n}^{\infty}\right)+E\left[\left(\mu_{n}^{\infty}\right)^{2}\left\|D_{n}^{\infty}\right\|^{2}\left(e_{n}^{\infty}\right)^{2}\right]
$$

Where $\operatorname{Tr}\left(Q_{n}^{\infty}\right)=\left[q_{n}^{\infty}\left(q_{n}^{\infty}\right)^{T}\right]$. By using equation (96) together with assumption $\mathrm{A} 4$ yields

$$
2 \mu_{n}^{\infty} \xi_{n}^{\infty}=\operatorname{Tr}\left(Q_{n}^{\infty}\right)+\overline{\left(\mu_{n}^{\infty}\right)^{2}} \operatorname{Tr}(\tilde{R})\left(\xi_{n}^{\infty}-\sigma_{\bar{v}_{n}^{\prime}}^{2}\right)
$$

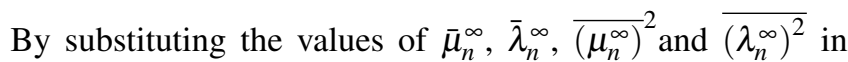
equation (97), we get the steady-state EMSE of the proposed algorithm.

$$
\xi_{n}^{\infty} \approx \frac{\mu \operatorname{Tr}(\tilde{R}) \sigma_{\bar{v}_{n}^{l}}^{2}}{2-\mu \operatorname{Tr}(\tilde{R})\left[1+\left(\gamma+\beta \sigma_{\bar{v}_{n}^{l}}^{2}\right) \sigma_{\bar{v}_{n}^{l}}^{2}\right]}+\operatorname{Tr}\left(\frac{Q_{n}^{\infty}}{2 \mu}\right)
$$

Since $q$ is assumed to be an i.i.d, therefore $\operatorname{Tr}\left(Q_{n}^{\infty}\right)=L \sigma_{q}^{2}$, so equation (98) will be reduced to

$$
\xi_{n(M N C L M S)}^{\infty} \approx \frac{\mu \operatorname{Tr}(\tilde{R}) \sigma_{\bar{v}_{n}^{l}}^{2}}{2-\mu \operatorname{Tr}(\tilde{R})\left[1+\left(\gamma+\beta \sigma_{\bar{v}_{n}^{l}}^{2}\right) \sigma_{\bar{v}_{n}^{l}}^{2}\right]}+\frac{L \sigma_{q}^{2}}{2 \mu}
$$

Where $L$ is the length of the filter.

\section{COMPUTATIONAL COMPLEXITY OF LINEAR EQUALIZER}

Computational cost is an important aspect of any algorithm. Higher computational cost can render an algorithm useless. A trade off between computational cost and the performance is possible, i.e. if the increased cost results in considerable performance gain, then higher cost can be ignored. In this section, we present the computational costs of few algorithms. As can be seen in table I, computational cost of the proposed algorithm is higher when compared to [4] but MNCLMS is for SISO- CDMA case, whereas, the proposed algorithm is for MIMO CDMA case. This algorithm can be used for runtime applications such as channel estimation, tracking and channel equalization. MIMO systems are being used in modern wireless standards.

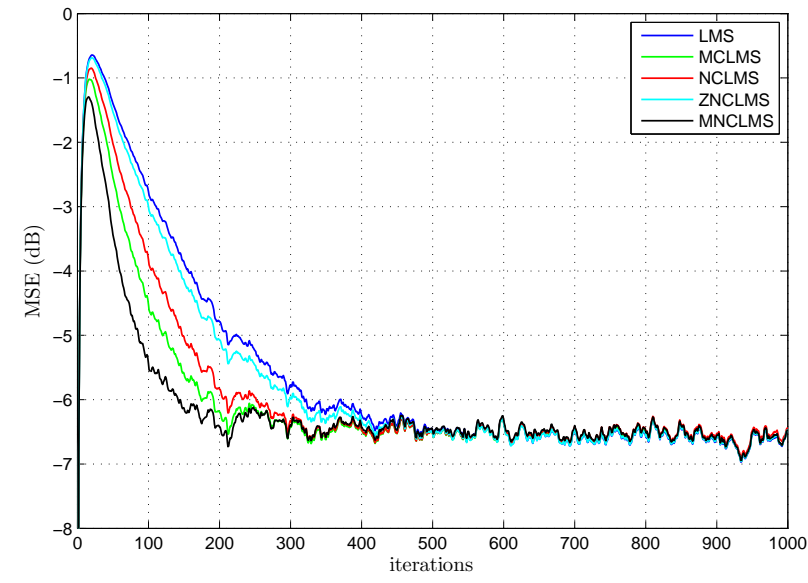

Fig. 1. MSE behavior for different algorithms in an AWGN environment with $K=10$ at $20 \mathrm{~dB}$

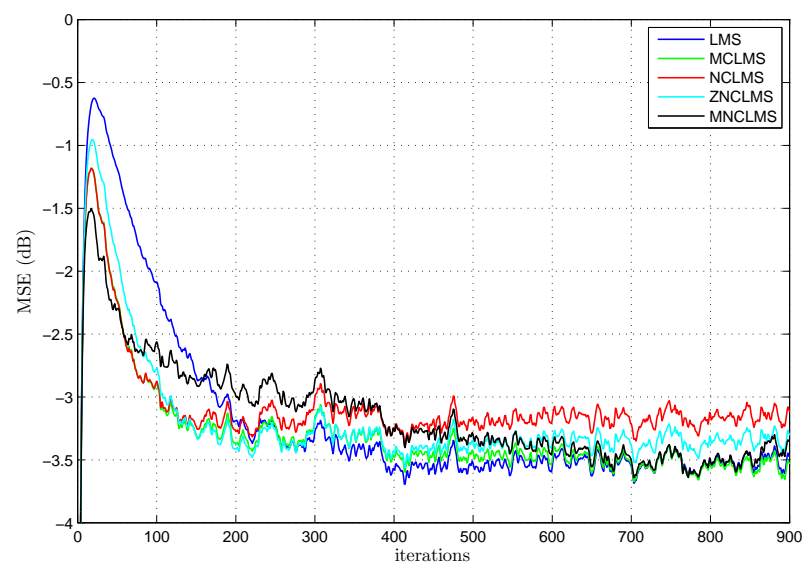

Fig. 2. MSE behavior for different algorithms in an AWGN environment with $K=20$ at $20 \mathrm{~dB}$

\section{Simulations}

Behavior of the step size of the MIMO-CDMA MNCLMS algorithm is shown in figure 3 for 10 subscribers. As can be seen, in the transient state, the MIMO-CDMA MNCLMS algorithm has the largest step-size value when compared to the other algorithms and, thus, yields the fastest convergence. Also, in the steady state, the step-size parameter of the MNCLMS algorithm was reduced to the smallest value amongst all algorithms. Same behavior is achieved for 20 subscribers as shown in figure 4

\section{A. Interference Cancellation in Rayleigh Fading Channel}

In this section, simulation results are presented to assess the performance of the MNCLMS algorithm for the MIMO CDMA LE case. The performance of the proposed MIMOCDMA MNCLMS algorithm is compared with the standard LMS, MCLMS noise constrained LMS and zero noise algorithms. The average MSE is the performance measure through which the algorithms are assessed. A $2 \times 2$ MIMO system is 


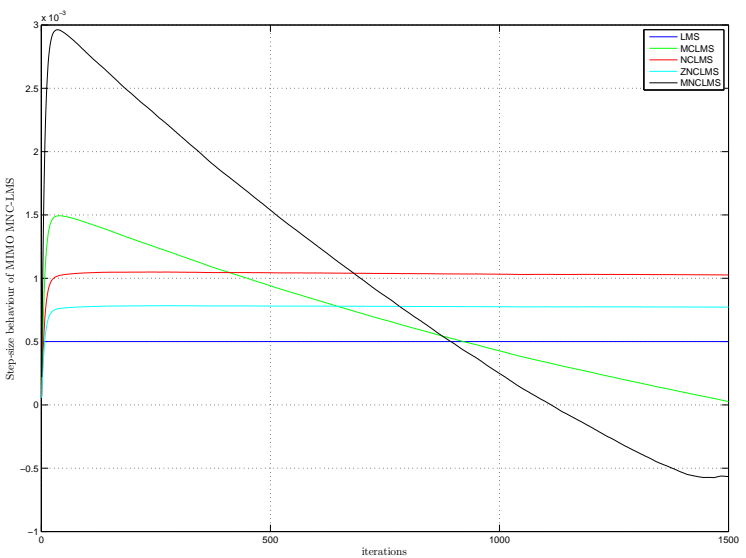

Fig. 3. Behavior of time-varying step size of the MNCLMS algorithm for $K=10$ at $20 \mathrm{~dB}$ SNR

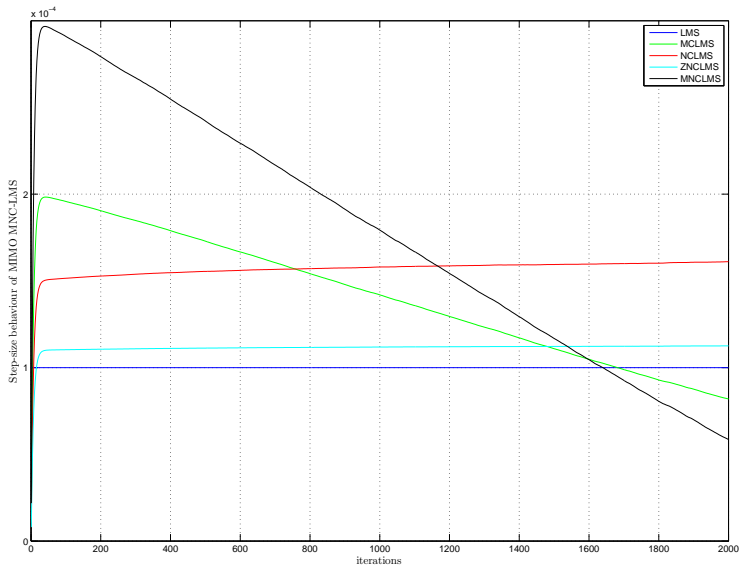

Fig. 4. Behavior of time-varying step size of the MNCLMS algorithm for $K=25$ at $20 \mathrm{~dB}$ SNR

considered here. Random signature sequences of length 31 and rectangular chip waveforms are used. The SNR is kept at 10 $\mathrm{dB}$ and $20 \mathrm{~dB}$ respectively.

The results of the comparison of the convergence speed of these algorithms for 10 and 25 subscribers, in a Rayleigh fading channel, are depicted, respectively, in Figure.5 and figure 6. In both cases, it can be seen that the MIMOCDMA MNCLMS algorithm converges faster than the rest of the algorithms. In the case of 10 subscribers, the proposed algorithm was able to achieve an MSE at around $-4.8 \mathrm{~dB}$ at around 200 iterations . In case of 25 subscribers, the proposed MNCLMS algorithm was able to achieve MSE at $-5.2 \mathrm{~dB}$ and in around 240 iterations.

\section{CONCLUSION}

In this paper a new constrained MIMO CDMA LMS type algorithm (MNCLMS) for multiuser wireless environment is presented. The MNCLMS algorithm may be refereed to as a generalized constrained adaptive algorithm which includes the MCLMS, the NCLMS and the ZNCLMS algorithms as

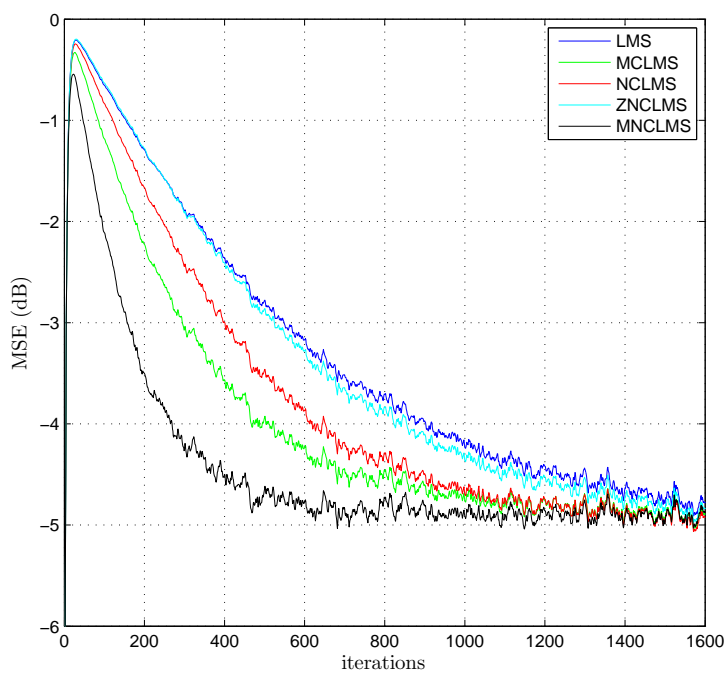

Fig. 5. MSE behavior for different algorithms in Rayleigh Fading environment with for $K=10$ at $10 \mathrm{~dB}$ SNR

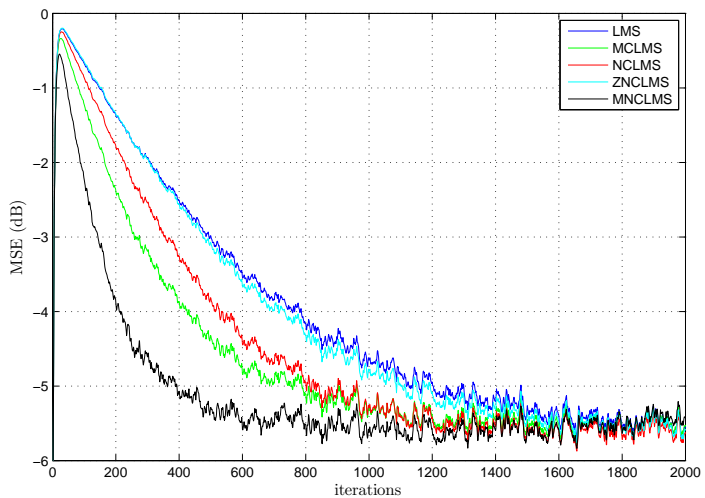

Fig. 6. MSE behavior for different algorithms in Rayleigh Fading environment for $K=20$ at $20 \mathrm{~dB}$ SNR

special cases. In our analysis, we have performed a thorough comparison of the proposed algorithm with a number of other constrained algorithms and it is shown that our algorithm has outperformed its competitors.

\section{REFERENCES}

[1] Yongbin Wei, Saul B Gelfand, and James V Krogmeier. Noise constrained $1 \mathrm{~ms}$ algorithm. In Acoustics, Speech, and Signal Processing, 1997. ICASSP-97., 1997 IEEE International Conference on, volume 3, pages 2353-2356. IEEE, 1997.

[2] RADU CIPRIAN Bilcu, Pauli Kuosmanen, and Corneliu Rusu. A noise constrained vs-lms algorithm. In EUROCOMM 2000. Information Systems for Enhanced Public Safety and Security. IEEE/AFCEA, pages 29-33. IEEE, 2000.

[3] SONG Woo-Jin. A complementary pair lms algorithm for adaptive filtering. IEICE TRANSACTIONS on Fundamentals of Electronics, Communications and Computer Sciences, 81(7):1493-1497, 1998.

[4] M Moinuddin, A Zerguine, and Asrar U. H. Sheikh. Multiple-Access Interference Plus Noise-Constrained Least Mean Square (MNCLMS) Algorithm for CDMA Systems. IEEE Transactions on Circuits and Systems I: Regular Papers, 55(9):2870-2883, October 2008.

[5] A.H. Sayed and T. Kailath. A state-space approach to adaptive rls filtering. Signal Processing Magazine, IEEE, 11(3):18-60, 1994. 
[6] Yongbin Wei, Saul B Gelfand, and James V Krogmeier. Noiseconstrained least mean squares algorithm. Signal Processing, IEEE Transactions on, 49(9):1961-1970, 2001.

[7] K. Mahmood, S.M. Asad, M. Moinuddin, A. Zerguine, and S. Paul. Statistical analysis of multiple access interference in rayleigh fading environment for mimo cdma systems. In Statistical Signal Processing (SSP), 2014 IEEE Workshop on, pages 412-415, June 2014.

[8] M Moinuddin, AUH Sheikh, A Zerguine, and M Deriche. A unified approach to ber analysis of synchronous downlink cdma systems with random signature sequences in fading channels with known channel phase. EURASIP Journal on Advances in Signal Processing, 2008(1):346465, 2008.

[9] Ali H Sayed. Adaptive filters. Wiley. com, 2008.

[10] Michael B Pursley. Performance evaluation for phase-coded spreadspectrum multiple-access communication. i-system analysis. IEEE Transactions on communications, 25:795-799, 1977.

[11] L Horowitz and K Senne. Performance advantage of complex $1 \mathrm{~ms}$ for controlling narrow-band adaptive arrays. Circuits and Systems, IEEE Transactions on, 28(6):562-576, 1981.

[12] R H Kwong and E W Johnston. A variable step size LMS algorithm. Signal Processing, IEEE Transactions on [see also Acoustics, Speech, and Signal Processing, IEEE Transactions on], 40(7):1633-1642, 1992.

[13] NEIL J Bershad. On error-saturation nonlinearities in lms adaptation. Acoustics, Speech and Signal Processing, IEEE Transactions on, 36(4):440-452, 1988.

[14] Neil J Bershad and MADELEINE Bonnet. Saturation effects in lms adaptive echo cancellation for binary data. Acoustics, Speech and Signal Processing, IEEE Transactions on, 38(10):1687-1696, 1990.

[15] Bernard Widrow, John M McCool, Michael G Larimore, and C Richard Johnson Jr. Stationary and nonstationary learning characteristics of the lms adaptive filter. Proceedings of the IEEE, 64(8):1151-1162, 1976.

[16] Nabil R Yousef and Ali H Sayed. A unified approach to the steadystate and tracking analyses of adaptive filters. Signal Processing, IEEE Transactions on, 49(2):314-324, 2001. 